\title{
The Ventral and Dorsal Default Mode Networks Are Dissociably Modulated by the Vividness and Valence of Imagined Events
}

\author{
${ }^{(D)}$ Sangil Lee, ${ }^{1 *}$ Trishala Parthasarathi, ${ }^{2 *}$ and Joseph W. Kable ${ }^{1}$ \\ ${ }^{1}$ Department of Psychology, University of Pennsylvania, Philadelphia, Pennsylvania 19104, and ${ }^{2}$ Department of Neuroscience, University of \\ Pennsylvania, Philadelphia, Pennsylvania 19104
}

Recent work has shown that the brain's default mode network (DMN) is active when people imagine the future. Here, we test in human participants (both sexes) whether future imagination can be decomposed into two dissociable psychological processes linked to different subcomponents of the DMN. While measuring brain activity with fMRI as subjects imagine future events, we manipulate the vividness of these events to modulate the demands for event construction, and we manipulate the valence of these events to modulate the demands for event evaluation. We found that one subcomponent of the DMN, the ventral DMN or medial temporal lobe (MTL) subsystem, responds to the vividness but not the valence of imagined events. In contrast, another subcomponent, the dorsal or core DMN, responds to the valence but not the vividness of imagined events. This separate modifiability of different subcomponents of the DMN by vividness and valence provides strong evidence for a neurocognitive dissociation between (1) the construction of novel, imagined events from individual components from memory and (2) the evaluation of these constructed events as desirable or undesirable.

Key words: DMN core; dorsal DMN; MTL DMN; valence; ventral DMN; vividness

\section{Significance Statement}

Previous work has suggested that imagination may depend on separate neural networks involved in the construction and evaluation of imagined future events. This study provides strong neural evidence for this dissociation by demonstrating that two components of the brain's default mode network (DMN) uniquely and specifically respond to different aspects of imagination. The vividness of imagined events modulates the ventral DMN, but not the dorsal DMN, while the valence of imagined events modulates the dorsal DMN, but not the ventral DMN. This supports the dissociable engagement of these subnetworks in constructing and evaluating imagined future events.

\section{Introduction}

Imagining the future can aid planning and help one act advantageously in the present. But what is the underlying cognitive architecture of imagination? Although imagination, like perception, can subjectively feel like a unitary experience, it may arise from the interaction of dissociable psychological processes. Here, we investigate the hypothesis that imagination consists of at least two distinct processes: a constructive process, by which a novel future event is mentally formed, often by combining specific aspects of past experience (Addis et al., 2007; Hassabis et al., 2007; Schacter et

\footnotetext{
Received May 22, 2020; revised Feb. 5, 2021; accepted Feb. 8, 2021

Author contributions: T.P. and J.W.K. designed research; T.P. performed research; S.L. and T.P. analyzed data; S.L., T.P., and J.W.K. wrote the paper.

This work was supported by the National Institute of Drug Abuse Grant R01 DA029149.

${ }^{*}$ S.L. and T.P. are joint first authors and are listed alphabetically.

The authors declare no competing financial interests.

Correspondence should be addressed to Joseph W. Kable at kable@psych.upenn.edu.

https://doi.org/10.1523/JNEUROSCI.1273-20.2021

Copyright $\odot 2021$ the authors
}

al., 2007), and an evaluative process, by which the imagined event is judged as positive or negative (D'Argembeau and Van Der Linden, 2004; Gilbert and Wilson, 2007; Sharot et al., 2007).

Because imagination is fundamentally an internal, subjective activity, studying its architecture can be difficult with behavioral data alone, and therefore many studies have turned to brain imaging. These studies have often focused on the default mode network (DMN), as envisioning and evaluating future events is proposed to be a key function of the DMN (Okuda et al., 2003; Addis et al., 2007; Sharot et al., 2007; Szpunar et al., 2007; Botzung et al., 2008). The DMN is one of the core networks reliably recovered from resting-state fMRI studies and includes the ventromedial prefrontal cortex (vmPFC), posterior cingulate cortex (PCC), and regions in the medial temporal and parietal lobes, such as hippocampus and precuneus (Greicius et al., 2004; Andrews-Hanna et al., 2007, 2010; Spreng et al., 2009; Raichle, 2015; Barnett et al., 2020).

Past research suggests that constructive and evaluative processes may engage different components of the DMN. Studies of 

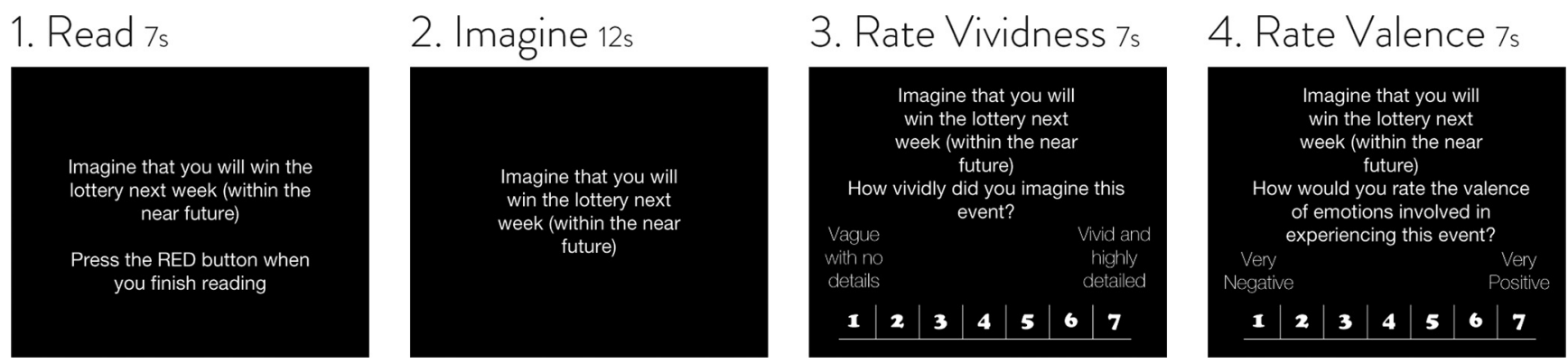

Figure 1. Experimental procedure of the task. Participants had up to $7 \mathrm{~s}$ to read the cue, $12 \mathrm{~s}$ to imagine, and up to $7 \mathrm{~s}$ each to rate the vividness and valence of the scenario.

"episodic simulation" that examined construction of potential future events have revealed activity in the hippocampus, parahippocampal gyrus, and retrosplenial cortex (Greicius et al., 2004; Addis et al., 2007, 2011; Hassabis and Maguire, 2007; Hassabis et al., 2007; Addis and Schacter, 2008). In contrast, activity in vmPFC is seen in tasks with an evaluative component. Activity in $\mathrm{VmPFC}$ is associated with the value of predicted future outcomes, and imagining positive events increases activity in vmPFC compared with imagining negative or control events (Sharot et al., 2007; D’Argembeau et al., 2008; Roy et al., 2012; Bartra et al., 2013; D’Argembeau, 2013; Benoit et al., 2014, 2019).

Resting-state functional connectivity studies also point to subdivisions of the DMN. Using seed-based resting-state functional connectivity, Andrews-Hanna et al. (2010) distinguished between a medial temporal lobe (MTL) subsystem, consisting of hippocampal, parahippocampal, restrosplenial, medial orbitofrontal, and posterior parietal cortex, and a DMN core, consisting of vmPFC and PCC [note that the vmPFC region in the DMN core was referred to as amPFC in Andrews-Hanna et al. (2010), while medial orbitofrontal cortex (OFC) region in the MTL subsystem was referred to as vmPFC; here, we follow the vmPFC and mOFC nomenclature]. Using independent components analysis, Shirer et al. (2012) proposed a similar distinction between a ventral DMN, which largely overlaps with the MTL subsystem, and a dorsal DMN, which largely overlaps with the DMN core.

Here, we constructed a strong test of the hypothesis that the DMN consists of dissociable constructive and evaluative networks involved in future imagination. We relied on the logic of separate modifiability (Sternberg, 2001), which supports stronger inferences regarding dissociations, by showing that two processes are differentially influenced by distinct factors within the same task. To modulate activity in brain regions engaged in constructive processes during imagination, we manipulated the vividness of imagined events, where vividness refers to the amount of detail or concreteness of the imagined event. To modulate activity in brain regions engaged in evaluative processes during imagination, we manipulated the valence of imagined events, where valence refers to the intensity of positive or negative emotions the imagined event invokes. If one component of the DMN is modulated by the vividness but not the valence of imagined events, while another component is modulated by valence but not vividness, this double dissociation would provide strong evidence for a functional division of the DMN associated with constructive versus evaluative processes.

\section{Materials and Methods}

All raw fMRI images and the behavioral responses are available online at openneuro.org (https://openneuro.org/datasets/ds002835). All analysis results, regressors, and regions of interest (ROIs) are available at OSF (https://dx.doi.org/10.17605/OSF.IO/CXHM4).

\section{Subjects}

Twenty-five participants ( 13 females, 12 males, average age $=24.9$ years, $\mathrm{SD}=4.6$ years) were recruited from the University of Pennsylvania and surrounding community. A sample size of 24 participants for analysis was determined in advance of the experiment. One participant was excluded for excessive head movement (shifts of at least $0.5 \mathrm{~mm}$ between $>5 \%$ of adjacent time points), which gave us 24 participants total for analysis. All participants were compensated for their time at $\$ 15$ per hour and provided consent before study procedures in accordance with the procedures of the Institutional Review Board of the University of Pennsylvania.

\section{Imaging acquisition and preprocessing}

Functional and anatomic images were collected using a 3T Siemens Trio scanner equipped with a 32-channel head coil. At the beginning of each session, high-resolution T1-weighted anatomic images were collected using an MPRAGE sequence (T1 $=1100 \mathrm{~ms} ; 160$ axial slices, $0.9375 \times$ $0.9375 \times 1.000 \mathrm{~mm} ; 192 \times 256$ matrix). T2*-weighted functional images were then collected using an EPI sequence (3-mm isotropic voxels, $64 \times 64$ matrix, 44 axial slices tilted $30^{\circ}$ from the AC-PC plane, $\mathrm{TR}=3000 \mathrm{~ms}, \mathrm{TE}=25 \mathrm{~ms}$ ), which gave us full coverage of all participants' brains. All participants completed four functional scans in each session, with each functional scan consisting of 181 images. At the end of the session, we acquired matched B0 fieldmap images (TR $=1000 \mathrm{~ms}$, $\mathrm{TE}=2.69$ and $5.27 \mathrm{~ms}$ ).

Brain imaging analysis was conducted with the FMRIB Software Library (FSL) using FSL FEAT (FMRIB fMRI Expert Analysis Tool) version 6.00 (Smith et al., 2004). Preprocessing included the following: (1) skull stripping of structural images with BET (FMRIB Brain Extract Tool); (2) motion correcting with MCFLIRT (FMRIB Linear Image Restoration Tool with Motion Correction); (3) spatial smoothing with a $9 \mathrm{~mm}$ full-width half-maximum Gaussian kernel; and (4) high-pass temporal filtering with a cutoff at $150 \mathrm{~s}(0.00667 \mathrm{~Hz})$. Registration and normalization were performed with FLIRT. Each functional image was registered to the participant's high-resolution brain-extracted structural image using boundary-based registration that simultaneously incorporates fieldmap-based geometric distortion and normalized to the FSL Montreal Neurologic Institute (MNI) template using affine transformations with 12 degrees of freedom.

\section{Experimental design and statistical analysis} Imagination task

All participants completed an imagination task in the scanner. Participants were asked to imagine scenarios and then rate the imagined scenarios on vividness and valence (Fig. 1). Sixteen scenarios were presented in each run and participants completed a total of four runs. The vividness and valence ratings were performed on a seven-point Likert scale. To assess vividness, participants were asked "How vividly did you imagine this event" with anchors of "Vague with no details" to "Vividly clear." To assess valence, participants were asked "How would you rate 
Table 1. Further characterization of vivid versus non-vivid and positive versus negative scenarios in separate online sample

\begin{tabular}{|c|c|c|c|c|c|c|c|c|c|c|c|c|}
\hline & Arousal & $\begin{array}{l}\text { Current } \\
\text { emotion }\end{array}$ & $\begin{array}{l}\text { Future } \\
\text { emotion }\end{array}$ & $\begin{array}{l}\text { Personal } \\
\text { importance }\end{array}$ & $\begin{array}{l}\text { Pre- } \\
\text { experience }\end{array}$ & $\begin{array}{l}\text { Self- } \\
\text { relevance }\end{array}$ & $\begin{array}{l}\text { Social } \\
\text { connection }\end{array}$ & $\begin{array}{l}\text { Temporal } \\
\text { distance }\end{array}$ & $\begin{array}{l}\text { Temporal } \\
\text { connection }\end{array}$ & $\begin{array}{l}\text { Visual } \\
\text { perspective }\end{array}$ & Valence & Vividness \\
\hline Non-vivid & 4.56 & 4.43 & 5.04 & 5.27 & 4.86 & 4.40 & 3.96 & 4.33 & 4.31 & 3.82 & 3.90 & 4.56 \\
\hline Vivid & 4.86 & 4.73 & 5.26 & 4.92 & 5.35 & 4.89 & 3.88 & 3.89 & 4.75 & 3.26 & 4.06 & 5.34 \\
\hline Negative & 4.42 & 4.92 & 5.41 & 5.02 & 4.83 & 4.35 & 3.01 & 4.45 & 4.06 & 3.62 & 2.11 & 4.86 \\
\hline Positive & 4.99 & 4.25 & 4.89 & 5.16 & 5.38 & 4.93 & 4.83 & 3.77 & 4.99 & 3.46 & 5.85 & 5.04 \\
\hline$t$ test $p$ & $0.004^{*}$ & $0.007^{*}$ & $0.032^{*}$ & 0.663 & $0.001^{*}$ & $0.010^{*}$ & $<0.001^{*}$ & 0.051 & $0.001^{*}$ & 0.356 & $<0.001^{*}$ & 0.364 \\
\hline
\end{tabular}

Average ratings for each of 14 different questions are provided and compared across non-vivid versus vivid scenarios and positive versus negative scenarios. Ratings were made of arousal, current emotion, future emotion, personal importance, pre-experience, self-relevance, social connection, subjective temporal distance, temporal connection, visual perspective, valence, and vividness. ${ }^{*} p<.05$.

the valence of emotions in this event" with anchors of "Very negative" to "Very positive."

Participants were given up to $7 \mathrm{~s}$ to read the cue, $12 \mathrm{~s}$ to imagine the scenario, up to $7 \mathrm{~s}$ to rate vividness, and up to $7 \mathrm{~s}$ to rate valence. The participant pressed a button indicating that the cue was read to start the imagination epoch. The imagination epoch was a fixed $12 \mathrm{~s}$ for all participants. Following imagination, participants were given up to $7 \mathrm{~s}$ to move a scale ranging from 1 to 7 to make their rating. If participants failed to submit a rating response within the allotted time, the last rating the participant had highlighted at that point was taken as their selection. Any time not used in any of the free response intervals was added to the intertrial interval, so that a new trial occurred every $33 \mathrm{~s}$.

\section{Scenarios}

The scenarios were selected for high or low vividness and for positive or negative valence. A list of 68 distinct scenarios was compiled from other studies that assessed vividness, valence, and other aspects of imagination, as well as a survey of Amazon Mechanical Turk (MTurk) respondents ( $n=411,199$ female, 212 male, average age $=30.1$ years, $\mathrm{SD}=11$ years) who were given broad categories of possible scenarios and asked to create their own. These scenarios were then rated in a separate study $(n=131,73$ female, 58 male, average age $=34.6$ years, $\mathrm{SD}=12$ years) on MTurk, with each participant rating the valence and vividness of 17 of the 68 scenarios. Based on these ratings, a final list of 32 scenarios was created by selecting the most and least vivid positive and negative scenarios. The final stimulus set included 8 scenarios in each of four conditions, vivid positive, vivid negative, non-vivid positive, and non-vivid negative.

To more exhaustively characterize the differences between vivid and non-vivid and positive and negative scenarios, we performed a further online survey $(n=391$, average age $=37.3$ years, $\mathrm{SD}=11.8$ years $)$. Online participants read each scenario and answered questions (shown below) about the imagined event. A different group of 32-34 participants answered for each of the following 12 different measures:

1. Arousal: What was your level of arousal in experiencing this event? $(1=$ not at all, $7=$ extremely $)$

2. Current emotion: How intense is the emotion felt at the time of imagining the event? $(1=$ not at all intense, $7=$ extremely intense $)$

3. Future emotion: How intense would your emotion be at the time when the future event takes place? $(1=$ not at all intense, $7=$ extremely intense)

4. Personal importance: What is the personal importance of this event? $(1=$ not important, 7 = extremely important $)$

5. Pre-experience: How much did you pre-experience the imagined event? (How much did you feel like you were actually there? $1=$ not at all, $7=$ completely)

6. Self-relevance: How relevant is the imagined event to you? ( $1=$ not at all relevant, $7=$ extremely relevant)

7. Social connection: How much did imagining this event make you feel connected to other people? $(1=$ not at all connected, $7=$ very connected)

8. Subjective temporal distance: How far away do you feel from the imagined future event? $(1=$ very close, 7 = very far $)$

9. Temporal connection: What is the perceived similarity of your current self to your self in the imagined future event? $(1=$ very different, 7 = exactly the same)
10. Visual perspective: What is your perspective when imagining this event? Are you actively participating (field) or simply observing (observer)? $(1=$ field, 7 = observer $)$

11. Valence: How would you rate the valence of emotions involved in experiencing this event? $(1=$ very negative, 7 = very positive $)$

12. Vividness: How vividly did you imagine this event? $(1=$ vague with no details, 7 = vivid and highly detailed)

The biggest difference between vivid and non-vivid scenarios was in vividness, but consistent with vividness affecting constructive processes, people were more likely to imagined vivid scenarios as active participants rather than observers (Table 1). The biggest difference between positive and negative scenarios was in valence, but consistent with valence affecting evaluative processes, people reported more arousal, less emotional intensity, a greater sense of social and temporal connectedness and more self-relevance for positive scenarios (Table 1). Both vivid and positive scenarios were associated with a greater feeling of being "actually there."

In the scanner, these 32 scenarios were repeated with both "near future" and "far future" prompts, where "near future" was defined as "within the next week" and "far future" was defined as "more than a year from now." This resulted in 64 unique scenario prompts, with each run containing 16 prompts. For the scanner participants, we conducted a repeated-measures ANOVA to ensure that there was a main effect of valence and vividness of the scenario on participants' judgments of valence and vividness.

\section{Imaging analyses}

The main goal of this study was to examine the dissociable roles of known DMN subcomponents in future imagination. One known subcomponent division comes from Shirer et al. (2012), who applied independent component analysis (ICA) to 15 participants' resting state data. Of the resulting 14 networks, one network was the ventral DMN and another was the dorsal DMN. The ROIs for these two subdivisions were downloaded from the author's website (http://findlab.stanford.edu/ functional_ROIs.html; Fig. 2, blue and red ROIs). Another known subcomponent division comes from Andrews-Hanna et al. (2010), who used seed-based functional connectivity procedures to define regions that comprised each subsystem. We created spherical regions of radius $=3$ voxels around the peak coordinates from that study to create ROIs for the DMN MTL subsystem (five spheres) and DMN core subsystem (two spheres; Fig. 2, green and yellow ROIs). While the two ways of dividing the DMN are not identical, there is substantial overlap between the ventral DMN and the DMN MTL subsystem, and between the dorsal DMN and the DMN core.

We used a general linear model to identify regions that were modulated by imagination vividness and valence. Our regressors included boxcar regressors for the read, imagine, rate vividness, and rate valence epochs, as well as categorical event modulators for the imagine epoch for the vividness (high vs low), valence (positive vs negative), and temporal distance (near vs far) of the imagined event, and parametric event modulators for the rate vividness and rate valence epochs (the participant's rating). In this analysis, we modeled the entire $12 \mathrm{~s}$ of the imagination epoch (another analysis described below examined subdivisions within the $12 \mathrm{~s}$ ). To assess whether the brain ROIs defined by Shirer et al. 

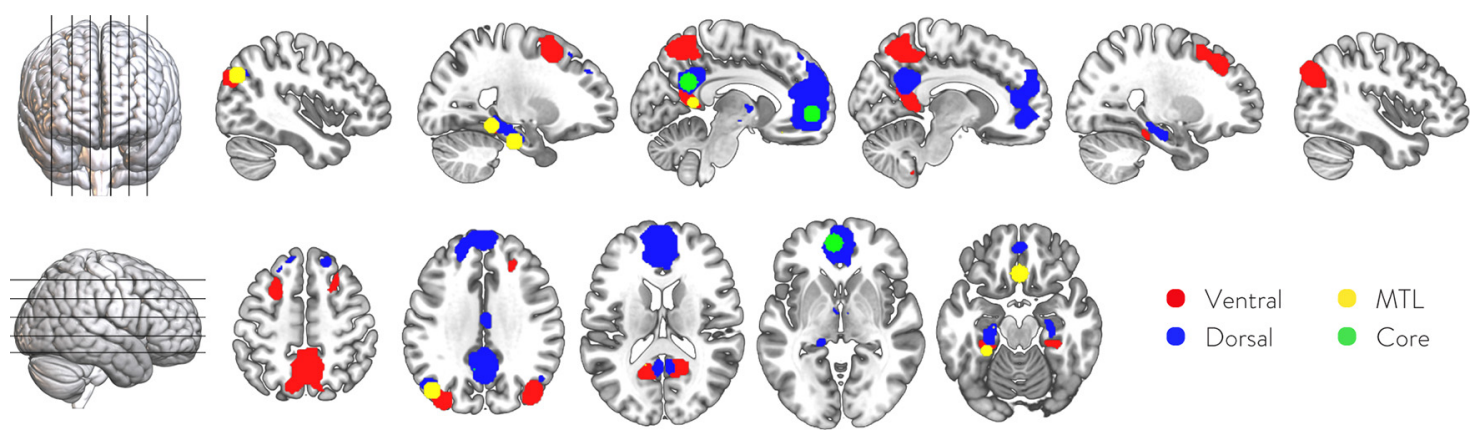

Figure 2. ROls from Shirer et al. (2012) consisting of ventral DMN and dorsal DMN and ROls from Andrews-Hanna et al. $(2007,2010)$ consisting of DMN MTL subsystem and DMN core.

(2012) and by Andrews-Hanna et al. (2010) were significantly modulated by vividness and valence, we calculated, for each of the four ROIs in each participant, the mean $\beta$ values of the vividness contrast regressor, the valence contrast regressor, and the difference between the two $\beta$ values averaged across all the voxels in each ROI mask. Then, these average $\beta$ values were t-tested against 0 at the group-level (across 24 participants) to assess whether the brain activity in each ROI was modulated by vividness or valence. In addition to testing modulation within ROIs, we also examined the same whole-brain group-level contrasts for significant regions via permutation (FSL randomize and threshold-free cluster enhancement) at corrected $p<0.05$.

To further assess the temporal specificity of the vividness and valence modulation, we divided the imagination period into three sections (early: $0-4 \mathrm{~s}$, middle: $4-8 \mathrm{~s}$, late: $8-12 \mathrm{~s}$ ) and assessed the degree of vividness and valence modulation in each time period. In the GLM, instead of one imagination epoch regressor, we included three separate imagination epoch boxcar regressors for the corresponding times. Also, instead of one vividness modulation regressor and one valence modulation regressor for the whole $12 \mathrm{~s}$, we included three modulation regressors, one for each of the separate time periods. To address the concern that the modulation regressors for the three epochs would be highly correlated and suffer from multicollinearity, we employed symmetric orthogonalization (also known as Lowdin orthogonalization; Löwdin, 1970) to orthogonalize the regressors for the three epochs to each other, while altering the regressors as little as possible. This is done by first preparing the regressors to be orthogonalized in a column-wise matrix (e.g., $\mathbf{M}$ ) and then performing singular value decomposition $(\mathbf{M}=\mathbf{U} \mathbf{\Sigma V})$. From the resulting three outputs (i.e., $\mathbf{U}, \boldsymbol{\Sigma}$, and $\mathbf{V}$ ), multiplying only the left and right singular vectors (i.e., $\mathbf{U}$ and $\mathbf{V}$ ) without the singular values (i.e., $\mathbf{\Sigma}$ ) results in a new matrix $\mathbf{U V}=\mathbf{M}^{*}$, which is the closest least-square approximation of $\mathbf{M}$ with orthogonal columns. The $\beta$ values for each of these three modulators were tested against zero at the group-level via permutation testing. The event regressors were not orthogonalized with respect to each other as only the effect of the modulation regressors were assessed in this analysis (symmetric orthogonalization takes linear sums of the original variables and hence does not alter the total variance explained by the event regressors). No testing was performed comparing the time periods.

\section{Results}

\section{Behavioral results}

Behavioral ratings confirmed that we had successfully manipulated the vividness and valence of imagined events (Fig. 3). There was a significant effect of vividness $\left(F_{(3,45)}=31.54\right.$, $p<0.001)$ as well as valence $\left(F_{(3,45)}=553.91, p<0.001\right)$ in a one-way ANOVA across the four conditions. Vividness ratings were significantly different between vivid $($ mean $=$ $5.32, \mathrm{SD}=0.36)$ and non-vivid (mean $=4.39, \mathrm{SD}=0.41)$ scenarios $\left(t_{(31)}=10.34, p<0.01\right.$, Cohen's $\left.d=1.54\right)$, but not between positive $($ mean $=4.9, \mathrm{SD}=0.59)$ and negative (mean $=4.8, \mathrm{SD}=0.63)$ scenarios $\left(t_{(31)}=0.84, p=0.42, d=0.14\right)$. Valence ratings were significantly different between positive (mean $=5.72, \mathrm{SD}=0.34)$ and negative $($ mean $=2.14, \mathrm{SD}=0.35)$ scenarios $\left(t_{(31)}=41.15, p<0.01, d=0.14\right)$. Valence ratings were also slightly more positive for vivid (mean $=4.04, \mathrm{SD}=1.90$ ) than nonvivid (mean $=3.82, \mathrm{SD}=1.80)$ scenarios $\left(t_{(31)}=2.29, \quad p=0.03\right.$, $d=1.95$ ). On average, participants' ratings of valence and vividness were weakly correlated at $r=0.14$. Note that each scenario was presented twice, once with a "in the near future" prompt and once with a "in the far future" prompt, but there were no behavioral effects of near versus far future.

\section{Imaging results}

The vividness and valence of imagined events modulated activity in distinct parts of the DMN (Fig. 4). To test our hypothesis regarding differential functional roles of previously defined DMN subcomponents, we first examined the division of the DMN into ventral and dorsal components, as described by Shirer et al. (2012). The ventral DMN was significantly modulated by vividness $\left(t_{(23)}=3.41, p=0.002\right.$, Cohen's $\left.d=0.69\right)$, but not by valence $\left(t_{(23)}=-0.65, p=0.52, d=0.13\right)$, and the effect of vividness was significantly larger than that of valence $\left(t_{(23)}=3.14\right.$, $p=0.005, d=0.64)$. The dorsal DMN was significantly modulated by valence $\left(t_{(23)}=2.71, p=0.01, d=0.55\right)$, but not vividness $\left(t_{(23)}=-0.90, p=0.38, d=0.19\right)$, and the effect of valence was 

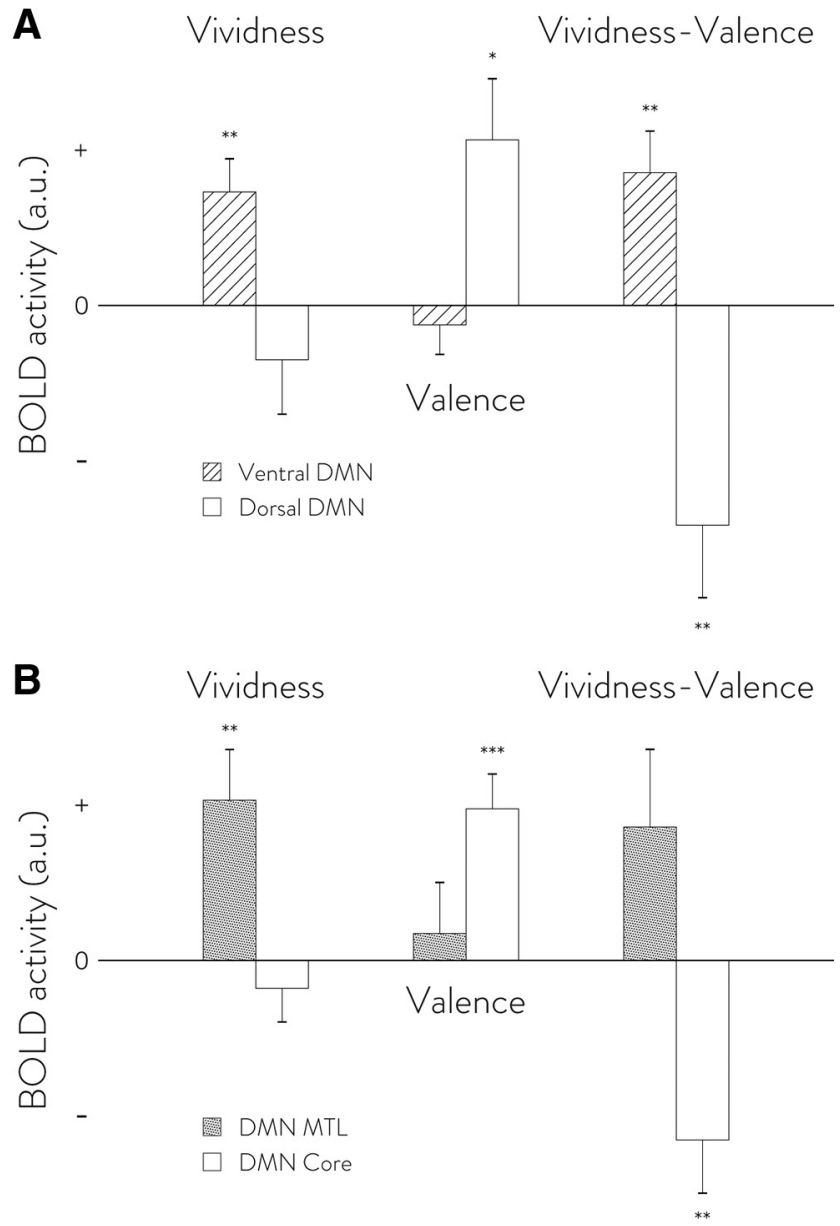

Figure 4. A, ROl results from the ventral and dorsal DMN, demonstrating that vividness but not valence significantly modulates the ventral DMN, while valence but not vividness significantly modulates the dorsal DMN. $\boldsymbol{B}$, ROI results from the DMN MTL subregions and DMN core, demonstrating that vividness but not valence significantly modulates the DMN MTL system, while valence but not vividness significantly modulates the DMN core $\left({ }^{*} p<0.05\right.$, $\left.{ }^{* *} p<0.01,{ }^{* *} p<0.001\right)$. Error bars indicate standard errors of the mean.

significantly larger than that of vividness $\left(t_{(23)}=-2.95, p=0.007\right.$, $d=0.60)$. In addition, vividness modulated the ventral DMN significantly more than the dorsal $\left(t_{(23)}=5.51, p<0.01, d=0.86\right)$, while valence modulated the dorsal DMN significantly more than the ventral $\left(t_{(23)}=-4.48, p<0.01, d=0.83\right)$.

We also observed the same dissociable modulations using the ROIs defined from peak coordinates of Andrews-Hanna et al. (2010; Fig. 4). The MTL subsystem of the DMN was significantly modulated by vividness $\left(t_{(23)}=3.13, p<0.01\right.$, Cohen's $\left.d=0.63\right)$, but not valence $\left(t_{(23)}=0.49, p=0.63, d=0.11\right)$, and the effect of vividness over valence was marginally significant $\left(t_{(23)}=1.75\right.$, $p=0.09, d=0.35)$. The DMN core was significantly modulated by valence $\left(t_{(23)}=4.52, p<0.01, d=0.91\right)$, but not vividness $\left(t_{(23)}=-0.78, p=0.44, d=0.16\right)$, and the effect of valence was significantly larger than that of vividness $\left(t_{(23)}=-3.45, p<0.01\right.$, $d=0.71$ ). In addition, vividness modulated the DMN MTL significantly more than the DMN core $\left(t_{(23)}=-3.84, p<0.01\right.$, $d=1.14$ ), while valence modulated the DMN core significantly more than the DMN MTL $\left(t_{(23)}=6.54, p<0.01, d=0.77\right)$.

Whole-brain analyses further confirmed the separate modulation of distinct neural regions by vividness and valence (Fig. 5). Across the entire imagination period, for trials with high compared with low vividness, there was increased activity in the left hippocampus, left dorsolateral PFC (dlPFC), and bilateral OFC.
For trials with positive compared with negative valence, there was increased activity in the vmPFC and striatum. Furthermore, activation in the vmPFC was modulated more by valence than by vividness.

When we further examined the temporal specificity of these effects within the imagination period, the effects of vividness were only present in the early phase of the imagination period, while those of valence were in both the early and middle phases. In the early phase (first $4 \mathrm{~s}$ ), more vivid scenarios elicited greater activity in the left dlPFC, bilateral hippocampus, and precuneus, while more positive scenarios elicited greater activity in the vmPFC and striatum. In the middle phase (second $4 \mathrm{~s}$ ), almost all of the effects of vividness are gone, while the effects of valence encompassed a larger area surrounding vmPFC and striatum. In the late phase (last $4 \mathrm{~s}$ ), there were no significant effects of vividness or valence (Table 2).

Although participants imagined each scenario twice, once in the near future and once in the far future, we did not observe any significant differences in activity between these two prompts, in either whole-brain or ROI analyses.

\section{Discussion}

Our results demonstrate a functional double dissociation within the DMN by showing the separate modifiability of different subcomponents of the DMN by different aspects of imagination. We manipulated the vividness of imagined events to engage constructive processes during imagination and the valence of imagined events to engage evaluative processes. Vividness, but not valence, modulated activity in the ventral DMN, or DMN MTL subsystem, including precuneus and MTL. (While the precuneus was not included in the original definition of the DMN MTL subsystem, recent results have found that precuneus clusters together with the MTL subsystem as well, see Barnett et al., 2020.) On the other hand, valence, but not vividness, modulated activity in the dorsal DMN, or DMN core, including the vmPFC. This basic pattern held in ROI analyses using two different sets of DMN ROIs, as well as in whole-brain analyses. Vividness-modulated activity also occurred exclusively early in the imagination period, while valence-modulated activity persisted later in the imagination period. These findings support functional specialization within the DMN, with the ventral DMN/MTL subsystem involved in the construction of imagined future events and the dorsal DMN/core involved in the evaluation of imagined future events.

As proposed by Sternberg (2001), the kind of separate modifiability demonstrated here between the ventral and dorsal DMN provides strong evidence for dissociable mental modules. The logic of separate modifiability is similar to that of the canonical double dissociation, although importantly focuses on dissociations between processes within the context of a single task, rather than on dissociations between tasks. Key to the inferential strength of separate modifiability is that different measures (in our case, neural activity in the ventral and dorsal DMN) are shown to be both sensitive and specific (i.e., responding to some manipulations but not others). Given the demonstration of separate modifiability, we can infer that the single complex process of imagination can be decomposed into component processes, putatively, construction and evaluation, which can each be uniquely influenced by the distinct factors of vividness and valence.

Our findings complement and expand on prior work regarding the role of the DMN in imagination. Many previous studies 


\section{Entire Period \\ $0-12 \mathrm{sec}$}

Vividness (High-Low)

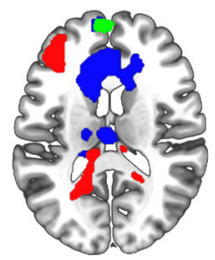

Vividness $>$ Valence
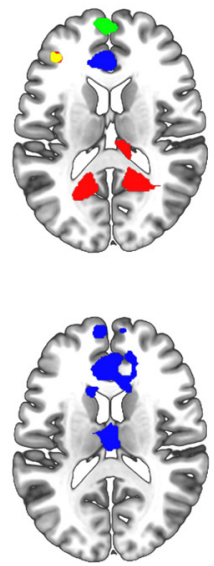
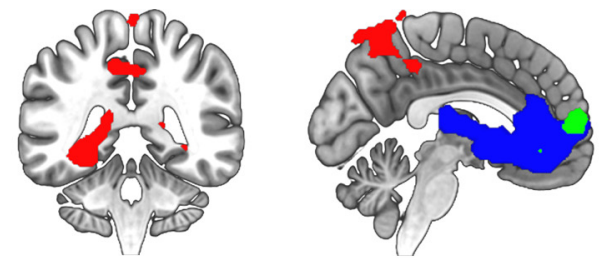

Valence (Pos-Neg)
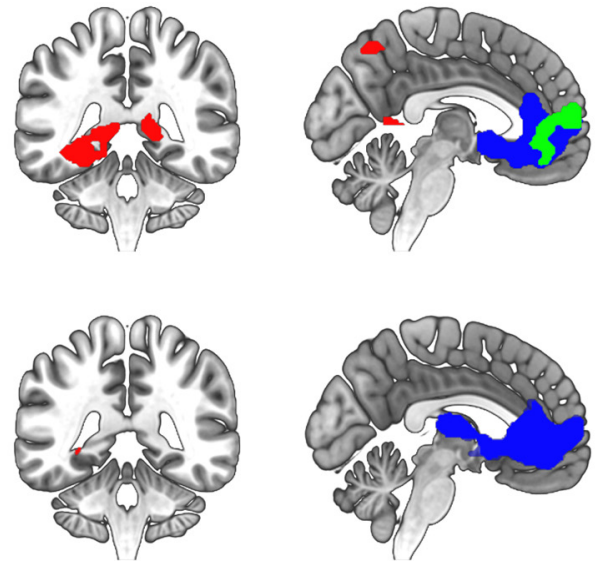

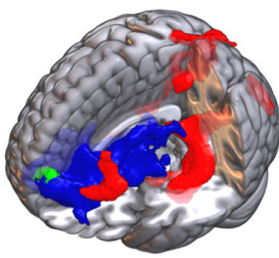

Valence $>$ Vividness
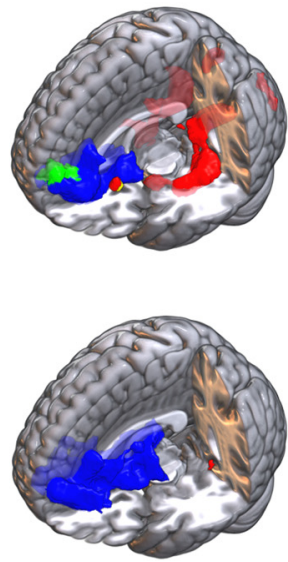

Figure 5. Whole-brain analysis of vividness and valence. Top panel shows the main effect of valence and vividness as well as their difference contrasts for the entire 12-s imagination period. The bottom two panels show the four effects for the early (first $4 \mathrm{~s}$ ) and middle (middle $4 \mathrm{~s}$ ) parts of the imagination period. There were no significant effects for the late (last $4 \mathrm{~s}$ ) part of the imagination period. Lists of the significant regions with their MNI coordinates are provided in Table 2.

Table 2. Regions significantly modulated by vividness or valence in the wholebrain analyses at different time points

\begin{tabular}{llrrr}
\hline Description & Epoch & $X$ & $Y$ & $Z$ \\
\hline $\begin{array}{l}\text { Vividness (high }>\text { low) } \\
\text { L hippocampus }\end{array}$ & Entire period (0-12 s) & -30 & -40 & -4 \\
& Early (0-4 s) & -32 & -38 & -10 \\
& Middle (4-8 s) & -30 & -44 & -2 \\
R hippocampus & Entire period (0-12 s) & 34 & -38 & -6 \\
& Early (0-4 s) & 14 & -48 & 12 \\
L DLPFC & Entire period (0-12 s) & -38 & 38 & 14 \\
& Early (0-4 s) & -36 & 36 & 14 \\
L precuneus & Entire period (0-12 s) & -10 & -58 & 56 \\
& Early (0-4 s) & -8 & -62 & 54 \\
L occipital middle & Entire period (0-12 s) & -44 & -84 & 28 \\
& Early (0-4 s) & -44 & -82 & 28 \\
R fusiform & Entire period (0-12 s) & 46 & -26 & -34 \\
Vividness $>$ valence & Early (0-4 s) & -38 & 36 & 14 \\
L DLPFC & Entire period (0-12 s) & -10 & 58 & 6 \\
Valence (Pos $>$ Neg) & Early (0-4 s) & 4 & 38 & -2 \\
vmPFC/VS & Middle (4-8 s) & -10 & 56 & 4 \\
& & & & \\
Valence $>$ vividness & Entire period (0-12 s) & -8 & 60 & 10 \\
vmPFC & Early (0-4 s) & -4 & 60 & 10 \\
m0FC & Entire period (0-12 s) & 4 & 34 & -8 \\
\hline
\end{tabular}

Regions such as bilateral hippocampus, bilateral OFC, left dIPFC, left precuneus, and right retrosplenial cortex were modulated by vividness, while vmPFC and VS were modulated by valence of imagined events.

have shown that imagination and other forms of "mental time travel" engage the DMN as a whole (Hassabis and Maguire, 2007; Hassabis et al., 2007; Schacter et al., 2007; Botzung et al., 2008). The DMN has greater metabolic activity at "rest," when participants are left undisturbed to generate spontaneous thought, than during different executive cognitive tasks (Bechara et al., 1994; Shulman et al., 1997; Raichle et al., 2001; Greicius et al., 2004; Andrews-Hanna et al., 2007, 2010; Spreng et al., 2009; Fox et al., 2015; Raichle, 2015). The DMN is also reliably activated when people engage in mental time travel in other ways, such as during tasks demanding autobiographic and social cognition, when people recall themselves in the past or think about someone else's mental perspective (Atance and O'Neill, 2001; Addis et al., 2007; Schacter et al., 2007; Sharot et al., 2007; D’Argembeau et al., 2008, 2014; Spreng et al., 2009, 2015; Tamir et al., 2016). With respect to valence, several previous studies have shown, as we do here, that vmPFC is more active when participants imagine positive compared with negative scenarios (D’Argembeau and Van Der Linden, 2004; Gilbert and Wilson, 2007; Sharot et al., 2007; Benoit et al., 2014, 2019). With respect to vividness, other studies have found that hippocampus is more active when people imagine future events in greater episodic detail (Addis and Schacter, 2008; Addis et al., 2011) or with a greater subjective experience of vividness (Thakral et al., 2020a,b).

Andrews-Hanna et al. (2010) first proposed that different subdivisions of the DMN serve different constructive and evaluative functions, with what they called the MTL subsystem of the DMN (including hippocampus) involved in the construction of mental scenes based on memory, and what they called the core DMN (including the vmPFC) involved in the affective evaluation of personal significance. The evidence to support this claim, however, was a single (as opposed to double) dissociation in which the MTL subsystem was more active when thinking about future than present events, while the DMN core was equally active in both conditions. A recent study investigating withinindividual networks has also supported the subsystem distinctions proposed by Andrews-Hanna and colleagues (DiNicola et al., 2020). However, other studies have observed different patterns of activity for thinking about the future versus the present, suggesting an alternative subdivision of the DMN into anterior 
and posterior components (Xu et al., 2016). Furthermore, subsequent studies of resting-state functional connectivity (Uddin et al., 2009; Sestieri et al., 2011; Xu et al., 2016), meta-analytic coactivation (Laird et al., 2009, 2013), or task fMRI dissociations have yielded yet additional proposals regarding DMN subdivisions (Leech et al., 2011; Sestieri et al., 2011; Whitfield-Gabrieli et al., 2011; Bado et al., 2014; Xu et al., 2016). Therefore, the separate modifiability by vividness and valence demonstrated here provides much stronger support for the distinction originally proposed by Andrews-Hanna and colleagues, between DMN components involved in constructive and evaluative processes during imagination.

Although we manipulated vividness to engage constructive processes and valence to engage evaluative processes, we do not expect that the activity modulations observed are necessarily unique to these specific features, as opposed to any set of features that would differentially engage construction versus evaluation. A broader set of potential features are seen in the more comprehensive ratings of our scenarios (Table 1). Vivid scenarios were also more likely to be imagined from a first person viewpoint, while positive scenarios were also higher in arousal, social and temporal connectedness, and self-relevance. Whether these features can be dissociated from each other, and whether some subset can be shown to be the primary driver of activity in the ventral or dorsal DMN, are important questions for future research. Regardless of the answer to these questions, the current results establish a clear dissociation between the roles of the ventral and dorsal DMN in constructive versus evaluative processes during imagination.

We also identified that the ventral striatum, which is not a part of the DMN, was more active when imagining positive compared with negative events. This is consistent with the involvement of the vmPFC and ventral striatum in evaluating outcomes and encoding predicted value during decision-making tasks (Kable and Glimcher, 2007; Bartra et al., 2013). Interestingly, we did not observe activation in other value regions such as PCC or central OFC, which have also been implicated in decision-making as well, with recent theories proposing that the OFC represents specific outcomes that are necessary for computing value and planning, rather than value itself, during choice tasks (Bechara et al., 2000; Ursu and Carter, 2005; Wallis, 2007).

Recent studies suggest that humans spend most of their time engaged in mental time travel, either remembering the past or imagining the future (Killingsworth and Gilbert, 2010). Yet we have very little formal understanding of the psychological processes involved in imagination. Our results suggest that the complex process of imagination, which might appear to be unitary, can in fact be decomposed into (at least) two dissociable mental processes, the construction of novel potential future events from components in memory and the evaluation of constructed events as desirable or undesirable. Neural measurements provided the key evidence for this dissociation, given the difficulty in constructing objective behavioral measures of imagination quality or ability. Thus, neuroscientific methods may prove critical to the further understanding of this central aspect of human subjective experience.

\section{References}

Addis DR, Schacter DL (2008) Constructive episodic simulation: temporal distance and detail of past and future events modulate hippocampal engagement. Hippocampus 18:227-237.
Addis DR, Wong AT, Schacter DL (2007) Remembering the past and imagining the future: common and distinct neural substrates during event construction and elaboration. Neuropsychologia 45:1363-1377.

Addis DR, Cheng T, P. Roberts R, Schacter DL (2011) Hippocampal contributions to the episodic simulation of specific and general future events. Hippocampus 21:1045-1052.

Andrews-Hanna JR, Snyder AZ, Vincent JL, Lustig C, Head D, Raichle ME, Buckner RL (2007) Disruption of large-scale brain systems in advanced aging. Neuron 56:924-935.

Andrews-Hanna JR, Reidler JS, Sepulcre J, Poulin R, Buckner RL (2010) Functional-anatomic fractionation of the brain's default network. Neuron 65:550-562.

Atance CM, O’Neill DK (2001) Episodic future thinking. Trends Cogn Sci 5:533-539.

Bado P, Engel A, Oliveira-Souza R, Bramati IE, Paiva FF, Basilio R, Sato JR, Tovar-Moll F, Moll J (2014) Functional dissociation of ventral frontal and dorsomedial default mode network components during resting state and emotional autobiographical recall. Hum Brain Mapp 35:3302-3313.

Barnett AJ, Reilly W, Dimsdale-Zucker H, Mizrak E, Reagh Z, Ranganath C (2020) Organization of cortico-hippocampal networks in the human brain. bioRxiv. doi: 10.1101/2020.06.09.142166.

Bartra O, McGuire JT, Kable JW (2013) The valuation system: a coordinatebased meta-analysis of BOLD fMRI experiments examining neural correlates of subjective value. Neuroimage 76:412-427.

Bechara A, Damasio AR, Damasio H, Anderson SW (1994) Insensitivity to future consequences following damage to human prefrontal cortex. Cognition 50:7-15.

Bechara A, Damasio H, Damasio AR (2000) Emotion, decision making and the orbitofrontal cortex. Cereb Cortex 10:295-307.

Benoit RG, Szpunar KK, Schacter DL (2014) Ventromedial prefrontal cortex supports affective future simulation by integrating distributed knowledge. Proc Natl Acad Sci USA 111:16550-16555.

Benoit RG, Paulus PC, Schacter DL (2019) Forming attitudes via neural activity supporting affective episodic simulations. Nat Commun 10:2215.

Botzung A, Denkova E, Manning L (2008) Experiencing past and future personal events: functional neuroimaging evidence on the neural bases of mental time travel. Brain Cogn 66:202-212.

D'Argembeau A (2013) On the role of the ventromedial prefrontal cortex in self-processing: the valuation hypothesis. Front Hum Neurosci 7:372.

D’Argembeau A, Van Der Linden M (2004) Phenomenal characteristics associated with projecting oneself back into the past and forward into the future: influence of valence and temporal distance. Conscious Cogn 13:844-858

D’Argembeau A, Xue G, Lu ZL, Van der Linden M, Bechara A (2008) Neural correlates of envisioning emotional events in the near and far future. Neuroimage 40:398-407.

D’Argembeau A, Cassol H, Phillips C, Balteau E, Salmon E, Van der Linden M (2014) Brains creating stories of selves: the neural basis of autobiographical reasoning. Soc Cogn Affect Neurosci 9:646-652.

DiNicola LM, Braga RM, Buckner RL (2020) Parallel distributed networks dissociate episodic and social functions within the individual. J Neurophysiol 123:1144-1179.

Fox KCR, Spreng RN, Ellamil M, Andrews-Hanna JR, Christoff K (2015) The wandering brain: meta-analysis of functional neuroimaging studies of mind-wandering and related spontaneous thought processes. Neuroimage 111:611-621.

Gilbert DT, Wilson TD (2007) Prospection: experiencing the future. Science 317:1351-1354

Greicius MD, Srivastava G, Reiss AL, Menon V (2004) Default-mode network activity distinguishes Alzheimer's disease from healthy aging: evidence from functional MRI. Proc Natl Acad Sci USA 101:4637-4642.

Hassabis D, Maguire EA (2007) Deconstructing episodic memory with construction. Trends Cogn Sci 11:299-306.

Hassabis D, Kumaran D, Maguire EA (2007) Using imagination to understand the neural basis of episodic memory. J Neurosci 27:14365-14374.

Kable JW, Glimcher PW (2007) The neural correlates of subjective value druing intertemporal choice. Nat Neurosci 10:1625-1633.

Killingsworth MA, Gilbert DT (2010) A wandering mind is an unhappy mind. Science 330:932-932.

Laird AR, Eickhoff SB, Li K, Robin DA, Glahn DC, Fox PT (2009) Investigating the functional heterogeneity of the default mode network 
using coordinate-based meta-analytic modeling. J Neurosci 29:1449614505.

Laird AR, Eickhoff SB, Rottschy C, Bzdok D, Ray KL, Fox PT (2013) Networks of task co-activations. Neuroimage 80:505-514.

Leech R, Kamourieh S, Beckmann CF, Sharp DJ (2011) Fractionating the default mode network: distinct contributions of the ventral and dorsal posterior cingulate cortex to cognitive control. J Neurosci 31:3217-3224.

Löwdin PO (1970) On the nonorthogonality problem. Adv Quantum Chem 5:185-199.

Okuda J, Fujii T, Ohtake H, Tsukiura T, Tanji K, Suzuki K, Kawashima R, Fukuda H, Itoh M, Yamadori A (2003) Thinking of the future and past: the roles of the frontal pole and the medial temporal lobes. Neuroimage 19:1369-1380.

Raichle ME (2015) The brain's default mode network. Annu Rev Neurosci 38:433-447.

Raichle ME, MacLeod AM, Snyder AZ, Powers WJ, Gusnard DA, Shulman GL (2001) A default mode of brain function. Proc Natl Acad Sci USA 98:676-682.

Roy M, Shohamy D, Wager TD (2012) Ventromedial prefrontal-subcortical systems and the generation of affective meaning. Trends Cogn Sci 16:147-156.

Schacter DL, Addis DR, Buckner RL (2007) Remembering the past to imagine the future: the prospective brain. Nat Rev Neurosci 8:657-661.

Sestieri C, Corbetta M, Romani GL, Shulman GL (2011) Episodic memory retrieval, parietal cortex, and the default mode network: functional and topographic analyses. J Neurosci 31:4407-4420.

Sharot T, Riccardi AM, Raio CM, Phelps EA (2007) Neural mechanisms mediating optimism bias. Nature 450:102-105.

Shirer WR, Ryali S, Rykhlevskaia E, Menon V, Greicius MD (2012) Decoding subject-driven cognitive states with whole-brain connectivity patterns. Cereb Cortex 22:158-165.

Shulman GL, Fiez JA, Corbetta M, Buckner RL, Miezin FM, Raichle ME, Petersen SE (1997) Common blood flow changes across visual tasks: II. Decreases in cerebral cortex. J Cogn Neurosci 9:648-663.

Smith SM, Jenkinson M, Woolrich MW, Beckmann CF, Behrens TEJ, Johansen-Berg H, Bannister PR, De Luca M, Drobnjak I, Flitney DE, Niazy RK, Saunders J, Vickers J, Zhang Y, De Stefano N, Brady JM,
Matthews PM (2004) Advances in functional and structural MR image analysis and implementation as FSL. Neuroimage 23:S208-S219.

Spreng RN, Mar RA, Kim ASN (2009) The common neural basis of autobiographical memory, prospection, navigation, theory of mind, and the default mode: a quantitative meta-analysis. J Cogn Neurosci 21:489-510.

Spreng RN, Gerlach KD, Turner GR, Schacter DL (2015) Autobiographical planning and the brain: activation and its modulation by qualitative features. J Cogn Neurosci 27:2147-2157.

Sternberg S (2001) Separate modifiability, mental modules, and the use of pure and composite measures to reveal them. Acta Psychol (Amst) 106:147-246.

Szpunar KK, Watson JM, McDermott KB (2007) Neural substrates of envisioning the future. Proc Natl Acad Sci USA 104:642-647.

Tamir DI, Bricker AB, Dodell-Feder D, Mitchell JP (2016) Reading fiction and reading minds: the role of simulation in the default network. Soc Cogn Affect Neurosci 11:215-224.

Thakral PP, Madore KP, Addis DR, Schacter DL (2020a) Reinstatement of event details during episodic simulation in the hippocampus. Cereb Cortex 30:2321-2337.

Thakral PP, Madore KP, Schacter DL (2020b) The core episodic simulation network dissociates as a function of subjective experience and objective content. Neuropsychologia 136:107263.

Uddin LQ, Kelly AMC, Biswal BB, Castellanos FX, Milham MP (2009) Functional connectivity of default mode network components: correlation, anticorrelation, and causality. Hum Brain Mapp 30:625-637.

Ursu S, Carter CS (2005) Outcome representations, counterfactual comparisons and the human orbitofrontal cortex: implications for neuroimaging studies of decision-making. Cogn Brain Res 23:51-60.

Wallis JD (2007) Orbitofrontal cortex and its contribution to decision-making. Annu Rev Neurosci 30:31-56.

Whitfield-Gabrieli S, Moran JM, Nieto-Castañón A, Triantafyllou C, Saxe R, Gabrieli JDE (2011) Associations and dissociations between default and self-reference networks in the human brain. Neuroimage 55:225-232.

Xu X, Yuan H, Lei X (2016) Activation and connectivity within the default mode network contribute independently to future-oriented thought. Sci Rep. 